\title{
PRIME ESSENTIAL RINGS
}

\author{
by B. J. GARDNER and P. N. STEWART
}

(Received 1st June 1989)

\begin{abstract}
A ring $R$ is prime essential if $R$ is semiprime and for each prime ideal $P$ of $R, P \cap I \neq 0$ whenever $I$ is a nonzero two-sided ideal of $R$. Examples of prime essential rings include rings of continuous functions and infinite products modulo infinite sums. We show that the class of prime essential rings is closed under many familiar operations; in particular, we consider polynomial rings, matix rings, fixed rings and skew group rings. Also, we explore the relationship between prime essential rings and special radical classes, and we demonstrate how prime essential rings can be used to construct radical classes which are not special.
\end{abstract}

1980 Mathematics subject classification (1985 Revision): 16A12, 16A21, 16A56.

\section{Introduction}

Throughout this paper the letters $R$ and $S$ will denote associative rings with identity and $A$ will denote an associative ring which may not have an identity. The ring $A$ is prime essential if $A$ is semiprime and each prime ideal of $A$ is essential as a two-sided ideal of $A$ (equivalently, $A$ is semiprime and every prime ideal of $A$ has nonzero intersection with each nonzero one-sided ideal of $A$ ). Prime essential rings were introduced by Rowen [23] and their importance stems from his Theorem 2 which states that every semiprime ring is a subdirect product of a prime essential ring and a ring which is an irredundant subdirect product of prime rings. Prime essential rings have been used to study commutative reduced rings (see [17], especially 3.12-3.15) and, as we shall see in the last section of this paper, they play a rôle in the study of special radical classes.

In the next section we show that the class of prime essential rings is closed under many familiar operations; for example, polynomial rings and matrix rings over prime essential rings are prime essential. Also, if $G$ is a finite group of automorphisms of a semiprime ring $R$ and $R$ has no $|G|$ torsion, then if one of $R^{G}, R, R G$ is prime essential, so too are the others. In the last section we explore the relationship between prime essential rings and special radical classes, and we demonstrate how prime essential rings can be used to construct radical classes which are not special.

The notation $I \triangleleft A$ means that $I$ is a two-sided ideal of $A, I \triangleleft^{\prime} A$ means that $I$ is a prime ideal of $A$. If $A$ is semiprime and $I \triangle A$, then $\{a \in A \mid a I=0\}=\{a \in A \mid I a=0\}$ is an ideal of $A$ which we shall denote by $I^{*}$. Let $A$ be a ring, $\kappa$ an infinite cardinal, $I$ an index set of cardinality $\kappa, P=\Pi\left\{A_{i} \mid A_{i}=A, i \in I\right\}$ and $J=\{p \in P \mid$ the cardinality of $\{i \in I \mid p(i) \neq 0\}$ is less than $\kappa\}$. The ring $P / J$ will be denoted by $A(\kappa)$. 
We shall make frequent use of the following proposition without making special reference to it.

Proposition 1. A ring $A$ is prime essential if and only if it is semiprime and no nonzero ideal of $A$ is a prime ring.

Proof. First assume that $A$ is prime essential and $0 \neq I \triangleleft A$. Then $I$ is not a prime ring because otherwise $I^{*} \triangleleft^{\prime} A$ which is not essential. Conversely, suppose that $P \triangleleft^{\prime} A$ and $P$ is not essential. Then $0 \neq P^{*}$ is isomorphic to an ideal of the prime ring $A / P$ and so $P^{*}$ is a prime ring.

We conclude this section with some examples of prime essential rings.

Example 1. Let $A_{i}, i \in I$, be an infinite family of semiprime rings, $P=\Pi\left\{A_{i} \mid i \in I\right\}$ and $J=\{p \in P \mid$ the cardinality of $\{i \in I \mid p(i) \neq 0\}$ is less than the cardinality of $I\}$. The ring $P / J$ is prime essential because its nonzero ideals are not prime rings. Note that when $A_{i}=A$ for all $i \in I$ we obtain $A(\kappa)$ where $\kappa$ is the cardinality of $I$.

Example 2. Let $X$ be a completely regular Hausdorff space with no isolated points. We will show that the ring $C(X)$ of real valued continuous functions on $X$ is prime essential. Let $0 \neq A \triangleleft C(X)$. There is an $f \in A$ and an $x \in X$ such that $f(x) \neq 0$. Since $f$ is continuous there is an open neighbourhood $\mathscr{U}$ of $x$ such that $z \in \mathscr{U}$ implies $f(z) \neq 0$. Because $x$ is not isolated there is a $w \in \mathscr{U}, w \neq x$. Since $X$ is Hausdorff $w$ and $x$ have disjoint neighborhoods $N_{1}$ and $N_{2}$ respectively, and since $X$ is completely regular there are $h, k \in C(X)$ such that $h(w)=1$ and $h(z)=0$ for all $z \in X \backslash N_{1}, k(x)=1$ and $k(z)=0$ for all $z \in X \backslash N_{2}$. It follows that $f h$ and $f k$ are nonzero elements of $A$ such that $(f h)(f k)=0$. Thus $A$ is not a prime ring and so $C(X)$ is prime essential. The special case of $C[0,1]$ is given by Rowen [23].

Example 3. Any Boolean ring which does not have an ideal isomorphic to $\mathbb{Z}_{2}$ is prime essential. These rings were used by Ryabukhin [21] to construct the first example of a hereditary supernilpotent radical class which is not special. Note that $\mathbb{Z}_{2}(\kappa)$, where $\kappa$ is any infinite cardinal, is an example of such a ring.

Example 4. Let $A_{1} \subseteq A_{2} \subseteq \ldots$ be a countably infinite ascending chain of semiprime rings. Let $S$ be the subring of $\Pi A_{i}$ consisting of all sequences $\bar{a}=\left(a_{1}, a_{2}, \ldots\right)$ for which there is an integer $n$, depending on $\bar{a}$, such that $a_{i}=a_{j}$ if $i \equiv j \bmod (n)$. The ring $S$ is prime essential and is isomorphic to a subring of the ring in Example 1 (when the index set $I$ in Example 1 is countable). This example is based on an idea of Snider [24, pp. 210, 211] where the $A_{i}$ form a strictly ascending chain of fields. The ring attributed to Leavitt in [14] is of this type where $A_{i}=\mathbb{Z}_{2}$ for all $i$.

Example 5. In this example we show that certain semigroup rings are prime 
essential. We require the following lemma which is adapted from [22, Lemma 8 and Lemma 9].

Lemma 1 (Ryabukhin). Let $S$ be a linearly ordered set with no greatest element, with least element $e$ and which is such that every interval $[x, y], x<y$, has cardinality $\kappa$. Then $S$ is a semi-group with multiplication defined by $x y=\max \{x, y\}$. Let $A$ be a nonzero semiprime ring.

(a) The semigroup ring $A(S)$ is a subdirect product of copies of $A$.

(b) $A(S)$ is semiprime.

(c) If $Q \triangleleft^{\prime} A(S)$, then $P=\{a \in A \mid a e \in Q\} \square^{\prime} A$ and $A(S) / Q \cong A / P$.

(d) If $I$ is a nonzero accessible subring of $A(S)$, then the cardinality of $I$ is at least $\kappa$.

Proof. (a) Since $A(S)$ is a strong semilattice sum of copies of $A$ this follows immediately from [6, Theorem 1.4]. Alternatively, if we define, for each $u \in S, I_{u}$ to be the ideal of $A(S)$ generated by elements of the form $a v-a u, a \in A, v<u$ and by elements of the form $b w, b \in A, w>u$, then it can be shown that $\bigcap\left\{I_{u}: u \in S\right\}=0$ and $A(S) / I_{u} \cong A$ for each $u \in S$.

(b) This follows immediately from (a).

(c) Let $Q \triangleleft^{\prime} A(S)$ and define $\phi: A \rightarrow A(S) / Q$ by $\phi(a)=a e+Q$. If $u \in S, a \in A$ and $u>e$, then $a u A(S)(a e-a u)=0$ and so $a u \in Q$ or $a e-a u \in Q$. Thus $\phi$ is onto and since $\phi$ is clearly a ring homomorphism, $A(S) / Q \cong A / P$ where $P=\operatorname{ker} \phi=\{a \in A \mid a e \in Q\}$.

(d) Let $I$ be a nonzero accessible subring of $A(S)$. This means that there are subrings $I_{1}, \ldots, I_{k}$ of $A(S)$ such that $0 \neq I=I_{1} \triangleleft I_{2} \triangleleft \cdots \triangleleft I_{k}=A(S)$. Since $A(S)$ is semiprime it follows from Andrunakievic's Lemma (see [8, Lemma 61]) that $I$ contains a nonzero ideal $J$ of $A(S)$. We now show that the cardinality of $J$ is at least $\kappa$.

Let $x=a_{1} u_{1}+\cdots+a_{k} u_{k} \in J, 0 \neq a_{i} \in A$ and $u_{i} \in S$ where $u_{1}<\cdots<u_{k}$. If $a=\sum_{i=1}^{k} a_{i} \neq 0$ then, since $A$ is semiprime, $a b \neq 0$ for some $b \in A$. Hence $a b u_{k}+a b v=x\left(b u_{k}+b v\right) \in J$ for all $v>u_{k}$. If $a=0$, then $c=\sum_{i=1}^{k-1} a_{i} \neq 0$ and the semiprimeness of $A$ guarantees that $c d \neq 0$ for some $d \in A$. Hence $c d u_{k-1}+a_{k} d u_{k}=x\left(d u_{k-1}\right) \in J$ where $a_{k} d=-c d \neq 0$. In any case there are $s, t \in S, s<t$ and nonzero $g, h \in A$ such that $g s+h t \in J$. Let $k \in A$ be such that $g k \neq 0$. Then for each $u$ in the interval $[s, t],(g s+h t) k u=g k u+h k t \in J$ and so the cardinality of $J$ is at least the cardinality of the interval $[s, t]$.

Let $A$ be any semiprime ring, let $\kappa$ be a cardinal number greater than the cardinality of $A$ and let $S$ be a linearly ordered set satisfying the hypotheses of the preceding lemma. We now verify that the semigroup ring $A(S)$ is prime essential. Let $Q \triangleleft^{\prime} A(S)$ and suppose that $I \triangleleft A(S)$ such that $Q \cap I=0$. Since $I \cong(I+Q) / Q$ we see from Lemma 1(c) that the cardinality of $I$ does not exceed the cardinality of $A$. Thus $I=0$ because of Lemma 1(d).

It easy to construct, for any cardinal $\kappa>1$, a linearly ordered set satisfying the hypotheses of Lemma 1. For example, the set $W(\kappa)$ of all finite words made from a (well-ordered) alphabet of cardinality $\kappa$, lexicographically ordered, is such a linearly 
ordered set. The corresponding prime essential semigroup rings $A(W(\kappa))$ will be used in the final section of the paper.

\section{Prime essential related rings}

Theorem 1. Let $I \triangleleft A$. (a) if $A$ is prime essential, so is $I$, (b) if $I$ is prime essential and an essential ideal of $A$, then $A$ is prime essential, (c) if $I$ and $A / I$ are prime essential, so is $A$, (d) if $0 \neq e=e^{2} \in A$ and $A$ is prime essential, then $e A e$ is prime essential.

Proof. (a) Since $A$ is semiprime it follows that $I$ is semiprime. Let $0 \neq J \triangleleft I$ and let $\hat{J}$ be the ideal of $A$ generated by $J$. Then $0 \neq(\widehat{J})^{3} \triangleleft J$ and since $A$ is prime essential, $\left(J^{3}\right.$ is not a prime ring. Because $\left(J^{3} \triangleleft J, J\right.$ is not a prime ring and so $I$ is prime essential.

(b) Since $I$ is semiprime, so is $A$. Let $0 \neq J \triangleleft A$. Then $0 \neq I \cap J \sqcup I$ and is consequently not a prime ring. Since $I \cap J \triangleleft J, J$ is not a prime ring. Hence $A$ is prime essential.

(c) Since $I$ and $A / I$ are semiprime, so is $A$. Let $0 \neq J \triangleleft A$. If $J \cap I=0$, then $J \cong(J+I) / I$ and so, since $A / I$ is prime essential, $J$ is not a prime ring. On the other hand, if $J \cap I \neq 0$, then $J \cap I$ is not a prime ring because it is an ideal of the prime essential ring $I$. Since $J \cap I \triangleleft J, J$ is not a prime ring. Thus $A$ is prime essential.

(d) Since $A$ is semiprime, so is $e A e$. Let $P \triangleleft^{\prime} e A e$. Then $P=e Q e$ for some $Q \square^{\prime} A$. If $I \triangleleft e A e$ and $P I=0$, then $(Q I)^{2}=0$. Since $A$ is semiprime and $Q$ is essential, $I=0$. Thus $P$ is essential in $e A e$ and so $e A e$ is prime essential.

The ring $S$ is a free centralizing extension of $R$ if $R \subseteq S, R$ and $S$ have the same identity, there is a subset $X \subseteq S$ such that $S$ is a free $R$-module with basis $X$ and $r x=x r$ for all $x \in X$ and $r \in R$.

Theorem 2. If $S$ is a semiprime free centralizing extension of a prime essential ring $R$, then $S$ is prime essential.

Proof. Let $Q \triangleleft^{\prime} S$. First we check that $Q \cap R \triangleleft^{\prime} R$. If $a, b \in R, a R b \subseteq Q$ and $s=\sum_{i=1}^{n} r_{i} x_{i} \in S, x_{i} \in X, r_{i} \in R$, then $a s b=\sum_{i=1}^{n} a r_{i} b x_{i} \in Q S \subseteq Q$. Since $Q \triangle^{\prime} S$ it follows that $Q \cap R \square^{\prime} R$. Since $R$ is prime essential, $(Q \cap R)^{*}=0$ and hence $Q^{*}=0$ because if $\sum_{i=1}^{n} r_{i} x_{i}, x_{i} \in X, r_{i} \in R$ is in $Q^{*}$, then $(Q \cap R) r_{i}=0$ for all $i=1, \ldots, n$. Thus $Q$ is essential in $S$ and the proof is complete.

Corollary 1. If $R$ is prime essential then so are matrix rings $M_{n}(R)$, polynomial rings $R[X]$ where $X$ is any set of indeterminates, and semiprime group rings $R G$.

Remark 1. The converse of Theorem 2 is true when $S$ is a finite free centralizing extension of $R$. To see this suppose that $S$ is a prime essential free centralizing extension of $R$ with a finite centralizing basis. Since $S$ is semiprime, $R$ is semiprime. Let $P \square^{\prime} R$. Then $P S \triangleleft S$ and $S / P S$ is a finite free centralizing extension of $R / P$. Thus it follows from 
[15, Corollary 4.14] that $S$ has prime ideals $Q_{1}, \ldots, Q_{m}$ such that $P S \subseteq N=\bigcap_{i=1}^{m} Q_{i}$ and $N^{t} \subseteq P S$ for some positive integer $t$. Since $Q_{i}^{*}=0$ for all $i=1, \ldots, m$ it follows that $P^{*}=0$, and so $R$ is prime essential.

However, the converse of Theorem 2 is not true in general: if $k$ is a field then $k$ embeds as the diagonal elements of $k\left(\boldsymbol{N}_{0}\right)$ and $k\left(\boldsymbol{\aleph}_{0}\right)$ is a prime essential free centralizing extension of $k$.

Theorem 3. Let $G$ be a finite group of automorphisms of $R$ such that $R$ has no $|G|$ torsion. The skew group ring $R G$ is prime essential if and only if $R$ is prime essential.

Proof. It follows from the Fisher-Montgomery Theorem [8] that $R$ is semiprime if and only if $R G$ is semiprime.

Assume that $R$ is prime essential and let $Q \square^{\prime} R G$. From [16, Lemma 4.2] we see that there are prime ideals $P_{1}, \ldots, P_{m}$ of $R$ such that $Q \cap R=\bigcap_{i=1}^{m} P_{i}$. Since each $P_{i}$ is essential, $Q \cap R$ is essential and so $(Q \cap R)^{*}=0$. If $s=\Sigma\left\{r_{g} g \mid g \in G\right\} \in R G$ and $Q s=0$, then $(Q \cap R) r_{g}=0$ for all $g \in G$. Thus $Q^{*}=0$ and so $R G$ is prime essential.

Now assume that $R G$ is prime essential and let $P \triangleleft^{\prime} R$. Choose $Q$ maximal in $\{I \triangleleft R G \mid I \cap R \subseteq P\}$. Then $Q \triangleleft^{\prime} R G$ and so $Q$ is essential as a right $R G$-submodule of $R G$. It follows from the essential Maschke's theorem [20, Lemma 1.2] that $Q$ is essential as a right $R$-submodule of $R G$ and so $Q \cap R$ is essential as a right ideal of $R$. Since $Q \cap R \subseteq P, P$ is essential and so $R$ is prime essential.

Remark 2. The argument in the above proof can be carried out in the more general setting of excellent extensions. See [19] for the required results about these extensions.

Remark 3. If $R$ is a ring graded by a finite group $G$, then the smash product, $R \# G$, is the free right and left $R$-module with basis $\left\{p_{g} \mid g \in G\right\}$ and multiplication determined by $\left(r p_{\theta}\right)\left(s p_{h}\right)=r s_{g h^{-1}} p_{h}$ where $s_{g h^{-1}}$ is the $g h^{-1}$ component of $s$. From [7, Theorem 3.5] we have $(R \# G) G \cong M_{n}(R)$ where $n=|G|$, so it follows from Corollary 1 , Remark 1 and Theorem 3 that if $R$ has no $|G|$ torsion then $R$ is prime essential if and only if $R \# G$ is prime essential.

Theorem 4. Let $G$ be a finite group of automorphisms of $R$ such that $R$ has no $|G|$ torsion. If $R$ is semiprime and the fixed ring $R^{G}$ is prime essential, then $R$ is prime essential. If $R$ is prime essential, so too is $R^{G}$.

Proof. Let $\hat{R}$ be the ring obtained by inverting $|G|$ in $R$; that is, $\hat{R}=R \otimes_{\mathrm{Z}} T$ where $T$ is the subring of the rationals which is generated by $|G|^{-1}$ and $\mathbb{Z}$ is the ring of rational integers. Clearly $R$ (respectively $R^{G}$ ) is prime essential (semiprime) if and only if $\hat{R}$ (respectively $\hat{R}^{G}$ ) is prime essential (semiprime). Thus we may assume that $|G|^{-1} \in R$.

Suppose that $R$ is semiprime and $R^{G}$ is prime essential. Let $Q \Delta^{\prime} R$ and set $\bar{Q}=\bigcap\{g(Q) \mid g \in G\}$. Then $\bar{Q}$ is $G$-invariant and $Q \cap R^{G}=\bar{Q} \cap R^{G}$. From [18, Proposition 4.2] we obtain a finite set $\left\{P_{1}, \ldots, P_{k}\right\}$ of prime ideals of $R^{G}$ such that $\bar{Q} \cap R^{G}=\bigcap_{i=1}^{k} P_{i}$. Since each $P_{i}$ is essential, $\bar{Q} \cap R^{G}$ is an essential ideal of $R^{G}$. If $I$ is a nonzero $G$-invariant left ideal of $R$, then it follows from the Bergman-Isaacs Theorem [4] that 
$I \cap R^{G} \neq 0$. Thus $\bar{Q}$ meets every nonzero $G$-invariant left ideal of $R$ nontrivially. The ring $R$ is naturally a left $R G$-module where the action of the skew group ring on $R$ is induced by left multiplication by elements of $R$ and the automorphism action by elements of $G$. The $R G$-submodules of $R$ are precisely the $G$-invariant left ideals of $R$ and so we have just shown that $\bar{Q}$ is essential as a left $R G$-submodule of $R$. By the essential Maschke's Theorem [20, Lemma 1.2] $\bar{Q}$ is essential as a left $R$-submodule of $R$ and so $\bar{Q}$, and hence $Q$, is an essential ideal of $R$.

Now assume that $R$ is prime essential. Then $R G$ is prime essential by Theorem 3 and so from Theorem 1 (d) $e(R G) e$ is prime essential where $e=|G|^{-1} \Sigma\{g \mid g \in G\}$. Since $R^{G} \cong e(R G) e, R^{G}$ is prime essential.

\section{Special radical classes}

For undefined radical theoretic terms see [8], [10], [25] or [26]. If $\mathscr{C}$ is a class of rings, $U(\mathscr{C})$ denotes the largest radical class $\mathscr{R}$ such that all rings in $\mathscr{C}$ are $\mathscr{R}$ semisimple. For hereditary classes $\mathscr{C}, U(\mathscr{C})$ is the class of all rings which have no nonzero homomorphic images in $\mathscr{C}$.

Theorem 5. The class $\mathscr{E}$ of prime essential rings is closed under finite, but not infinite, subdirect products. The subdirect product closure of $\mathscr{E}$ is the class of $U(\mathscr{E})$ semisimple rings.

Proof. It follows from Theorem 1(a) and (c) that the class of prime essential rings is closed under finite subdirect products.

Let $\mathbb{Z}$ be the ring of integers and $B=\mathbb{Z}\left(\aleph_{0}\right)$. Let $\mathscr{V}$ be the variety of associative rings generated by $B$. Since the subring of $B$ generated by the identity is $\mathbb{Z}, \mathbb{Z} \in \mathscr{V}$. The polynomial ring $\mathbb{Z}[x]$ is subdirect product of copies of $\mathbb{Z}$, so $\mathbb{Z}[x] \in \mathscr{V}$, and by induction $\mathbb{Z}\left[x_{1}, \ldots, x_{n}\right] \in \mathscr{V}$ for any finite set of commuting indeterminates $\left\{x_{1}, \ldots, x_{n}\right\}$. Thus all finitely generated commutative rings are in $\mathscr{V}$ and so $\mathscr{V}$ is the variety of all commutative rings.

It follows from Burris [5] that there is an infinite cardinal $\kappa$ such that the free ring in $\mathscr{V}$ on $\kappa$ generators is a subdirect product of copies of $B$. Since $B$ is prime essential and this free ring, which is just the free commutative ring on $\kappa$ generators, is not, the class $\mathscr{E}$ is not closed under arbitrary subdirect products.

The rest of the theorem follows from [2, Theorem 1].

A hereditary radical class $\mathscr{R}$ which contains all nilpotent rings is special if every $\mathscr{R}$ semisimple ring is a subdirect product of prime $\mathscr{R}$ semisimple rings.

Theorem 6. $A$ hereditary radical class $\mathscr{R}$ which contains all nilpotent rings is special if and only if every prime essential $\mathscr{R}$ semi-simple ring is a subdirect product of prime $\mathscr{R}$ semisimple rings.

Proof. One direction is obvious. For the other, suppose that $A$ is $\mathscr{R}$ semisimple. Let 
$\Delta=\bigcap\left\{P \triangleleft^{\prime} A \mid P^{*} \neq 0\right\}$ (we take $\Delta=A$ if $P^{*}=0$ for all $P \triangleleft^{\prime} A$ ). If $P^{*} \neq 0$, then $A / P$ is $\mathscr{R}$ semisimple because it has an essential ideal $\left(P^{*}+P\right) / P$ which is isomorphic to the $\mathscr{R}$ semisimple ring $P^{*}$. We are trying to show that $A$ is a subdirect product of prime $\mathscr{R}$ semisimple rings, and this is clearly the case if $\Delta=0$. We now assume that $\Delta \neq 0$. Rowen [23] has shown that $A / \Delta^{*}$ is prime essential and $A / \Delta^{*}$ is $\mathscr{R}$ semisimple because it has an essential ideal which is isomorphic to the $\mathscr{R}$ semisimple ring $\Delta$. Since $A$ is semiprime, $\Delta \cap \Delta^{*}=0$ and the result follows.

If $\mathscr{C}$ is a class of rings the smallest radical class containing $\mathscr{C}$ will be denoted by $L(\mathscr{C})$, and if $\mathscr{R}_{1}$ and $\mathscr{R}_{2}$ are radical classes with $\mathscr{R}_{1} \subseteq \mathscr{R}_{2}$, the interval of radical classes $\mathscr{R}$ such that $\mathscr{R}_{1} \subseteq \mathscr{R} \subseteq \mathscr{R}_{2}$ will be denoted by $\left[\mathscr{R}_{1}, \mathscr{R}_{2}\right]$.

Lemma 2. Let $\mathscr{R}$ be a radical class, $\mathscr{C}$ a class of $\mathscr{R}$ semisimple rings and $B$ a ring. If $B$ is a subdirect product of rings in $\mathscr{C}, B$ is $L(\mathscr{C})$ semisimple and every prime homomorphic image of $B$ is in $L(\mathscr{C})$ then $L(\mathscr{R} \cup \mathscr{C}) \subseteq U(\{B\})$ and no radical class in $[L(\mathscr{R} \cup \mathscr{C}), U(\{B\})]$ is special.

Proof. Since $B$ is a subdirect product of rings in $\mathscr{C}$ which are $\mathscr{R}$ semisimple, $B$ is $\mathscr{R}$ semisimple. Since $B$ is also $L(\mathscr{C})$ semisimple it must be $L \mathscr{R} \cup \mathscr{C}$ ) semisimple and so $L(\mathscr{R} \cup \mathscr{C}) \subseteq U(\{B\})$. Now if $\mathscr{R}_{1}$ is a radical class in $[L(\mathscr{R} \cup \mathscr{C}), U\{B\}]$, then $B$ is $\mathscr{R}_{1}$ semisimple but all prime homomorphic images of $B$ are in $\mathscr{R}_{1}$. Hence $\mathscr{R}_{1}$ is not special.

Theorem 7. Let $\mathscr{R}$ be a radical class and let $A$ be a nonzero semiprime $\mathscr{R}$ semisimple ring. Then for any infinite cardinal $\kappa$ larger than the cardinality of $A, L(\mathscr{R} \cup\{A\}) \subseteq U(\{A(W(\kappa))\})$ and no radical class in $[L(\mathscr{R} \cup\{A\}), U(\{A(W(\kappa))\})]$ is special.

Proof. It follows from Lemma $1(\mathrm{~d})$ that $A(W(\kappa))$ is $L(\{A\})$ semisimple and so Lemma 1(a), (b) and (c) show that Lemma 2 applies.

Remark 4. We note that for every radical class $\mathscr{R}$, other than the class of all rings, there do exist nonzero semiprime $\mathscr{R}$ semisimple rings, and hence prime essential $\mathscr{R}$ semisimple rings. To see this, suppose that $A$ is a nonzero $\mathscr{R}$ semisimple ring. If $A$ is not semiprime, then, since semisimple classes are hereditary, either $\mathbb{Z}^{0}$ (the zero ring on the integers) or $\mathbb{Z}_{p}^{0}$ (the zero ring on the integers modulo $p, p$ prime) is $\mathscr{R}$ semisimple. Let $B=\mathbb{Z}$ or $\mathbb{Z}_{p}$, so that the zero ring on $B$ is $\mathscr{R}$ semisimple. Since semisimple classes are closed under extensions it follows by induction that $(x B[x]) /\left(x^{k}\right)$ is $\mathscr{R}$ semisimple for all integers $k \geqq 2$, where $\left(x^{k}\right)$ denotes the ideal of $B[x]$ generated by $x^{k}$. Now because semisimple classes are closed under subdirect products, the semiprime ring $x B[x]$ is $\mathscr{R}$ semisimple.

Remark 5. If $\mathscr{R}$ is supernilpotent (that is, contains all nilpotent rings), then certainly all the radical classes in the interval $[L(\mathscr{R} \cup\{A\}), U(\{A(W(\kappa))\})]$ are also supernilpotent. 
Also, if $\mathscr{R}$ is hereditary, the interval above contains hereditary radical classes; for example, $L(\mathscr{R} \cup \mathscr{H})$ where $\mathscr{H}$ is the class of accessible subrings of $A$.

Theorem 8. Let $\mathscr{R}$ be a radical class and $\mathscr{C}$ a class of $\mathscr{R}$ semisimple rings such that (a) every semiprime homomorphic image of a ring in $\mathscr{C}$ is prime and (b) there is a prime essential ring $E$ such that every prime homomorphic image of $E$ is in $\mathscr{C}$. Then $L(\mathscr{R} \cup \mathscr{C}) \subseteq U(\{E\})$ and no radical class in $[L(\mathscr{R} \cup \mathscr{C}), U(\{E\})]$ is special.

Proof. It follows from (b) that $E$ is a subdirect product of rings in $\mathscr{C}$ and that every prime homomorphic image of $E$ is in $L(\mathscr{C})$. Also, every accessible subring of $E$ is prime essential and so not a homomorphic image of a ring in $\mathscr{C}$ by (a). Hence $E$ is $L(\mathscr{C})$ semisimple and so the result follows from Lemma 2.

Example 6. Let $\mathscr{C}$ be the class of prime homomorphic images of the ring $C(X)$ described in Example 2. Then $\mathscr{C}$ satisfies condition (a) of the theorem [13, Theorem 2.4] and $C(X)$ satisfies the conditions required of $E$ in (b). It follows, in particular, that $U(\{C(X)\})$ is not special.

Example 7. Let $B$ be a prime von Neumann regular ring which satisfies general comparability (see [12, Chapter 9]). Then any product $P$ of copies of $B$ also satisfies general comparability [12, page 83] and so the ideals in all prime homomorphic images of $P$ are linearly ordered [12, Theorem 8.20]. Since all homomorphic images of regular rings with linearly ordered ideals are prime, the class $\mathscr{C}$ of prime homomorphic images of products of $B$ satisfies condition (a) of the theorem. The ring $B(\kappa)$, where $\kappa$ is any infinite cardinal, satisfies condition (b). Examples of von Neumann regular rings which satisfy general comparability include full endomorphism rings of vector spaces over division rings and, more generally, all prime, regular, self-injective rings [12, Corollary 9.16].

Let $A$ be a ring, $I$ an index set and $A^{I}=\Pi\left\{A_{i} \mid A_{i}=A, i \in I\right\}$. An ultrapower of $A$ is a ring of the form $A^{I} / B$ where $B=\left\{p \in A^{I} \mid\{i \mid p(i)=0\} \in \mathscr{T}\right\}$ and $\mathscr{T}$ is an ultrafilter of subsets of $I$.

Corollary 2. Let $\mathscr{R}$ be a radical class and $\mathscr{C}$ a class of $\mathscr{R}$ semisimple rings such that (a) every semiprime homomorphic of a ring in $\mathscr{C}$ is a prime ring in $\mathscr{C}$ and (b) there is a prime ring $P \in \mathscr{C}$ such that all ultrapowers of $P$ are in $\mathscr{C}$. Then for each infinite cardinal $\kappa$, $L \mathscr{R} \cup \mathscr{C}) \subseteq U(\{P(\kappa)\})$ and no radical class in $[L \mathscr{R} \cup \mathscr{C}), U(\{P(\kappa)\})]$ is special.

Proof. It suffices to show that for each infinite cardinal $\kappa$, all prime homomorphic images of $P(\kappa)$ are in $\mathscr{C}$. Let $Q$ be a minimal prime ideal of $P(\kappa)$. Then the inverse image of $Q$ in $\Pi\left\{A_{i} \mid A_{i}=P, i \in I\right\}$, where the cardinality of $I$ is $\kappa$, is a minimal prime. Thus $P(\kappa) / Q$ is an ultrapower of $P[11$, Proposition 3.3]. It then follows from (b) that $P(\kappa) / Q \in \mathscr{C}$ and so (a) implies that all prime homomorphic images of $P(\kappa)$ are in $\mathscr{C}$.

Example 8. Let $\mathscr{V}$ be a proper variety of associative rings and let $\mathscr{C}$ be the class of 
simple prime rings in $\mathscr{V}$. Clearly $\mathscr{C}$ satisfies condition (a) of the corollary and, if $\mathscr{C} \neq \phi$, (b) is satisfied by every ring in $\mathscr{C}[1$, Theorems $2 \mathrm{~A}$ and $2 \mathrm{~B}]$.

For a P.I. ring $A$, let $d(A)=\inf \{k \mid A$ satisfies the standard identity of degree $2 k\}$.

Theorem 9. Let $\mathscr{E}$ be the class of prime essential rings.

(a) $U(\mathscr{E})$ is not special.

(b) [Ryabukhin, 21]. $U(\mathscr{D})$ is not special where $\mathscr{D}$ is the class of all rings which are subdirect products of copies of $\mathbb{Z}_{2}$ but which have no ideals isomorphic to $\mathbb{Z}_{2}$.

(c) [van Leeuwen and Jenkins, 14]. For $n>1, U\left(\mathscr{D}_{n} \cup \mathscr{F}\right)$ is not special where $\mathscr{D}_{n}$ is the class of all rings which are subdirect products of copies of $M_{n}\left(\mathbb{Z}_{2}\right)$ but which have no ideals isomorphic to $M_{n}\left(\mathbb{Z}_{2}\right)$ and $\mathscr{F}$ is the class of all fields.

(d) [Ryabukhin, 22]. Let $\kappa$ be an infinite cardinal and $\mathscr{S}$ the class of all rings of cardinality not exceeding $\kappa$. Then $L(\mathscr{P})$ is not special.

(e) [Beidar and Salavová, 3]. For $n \geqq 1, U\left(\mathscr{E}_{n}\right)$ is not special where $\mathscr{E}_{n}=\{A \in \mathscr{E} \mid d(A)=n\}$.

Proof. (a) and (b). These follow immediately from Theorem 7 with $\mathscr{R}=\{0\}$ and $A=\mathbb{Z}_{2}$ or from Theorem 8 with $\mathscr{R}=\{0\}, \mathscr{C}=\left\{\mathbb{Z}_{2}\right\}$ and $E=Z_{2}\left(\aleph_{0}\right)$. All prime homomorphic images of $E$ are in $\mathscr{C}$ because $\mathbb{Z}_{2}$ is the only prime Boolean ring.

(c) As above we can use either Theorem 7 or Theorem 8 , but with $M_{n}\left(\mathbb{Z}_{2}\right)$ in place of $\mathbb{Z}_{2}$. The only prime homomorphic images of $E=M_{n}\left(\mathbb{Z}_{2}\right)\left(\aleph_{0}\right) \cong M_{n}\left(\mathbb{Z}_{2}\left(\aleph_{0}\right)\right)$ are of the form $E / M_{n}(I)$ where $I \triangleleft^{\prime} \mathbb{Z}_{2}\left(\aleph_{0}\right)$ and hence $\mathbb{Z}_{2}\left(\aleph_{0}\right) / I \cong \mathbb{Z}_{2}$. So these prime homomorphic images are all isomorphic to $M_{n}\left(\mathbb{Z}_{2}\right)$.

(d) Let $\mathscr{R}=\{0\}$ and $A=\mathbb{Z}_{2}$. In view of Lemma $1(\mathrm{~d}), L(\mathscr{S}) \subseteq U(\{A(W(\alpha))\})$ where $\alpha$ is any cardinal greater than $\kappa$. Thus the result follows from Theorem 7 . The result also follows from Theorem 8 with $\mathscr{R}=\{0\}, \mathscr{C}=\left\{\mathbb{Z}_{2}\right\}$ and $E=\mathbb{Z}_{2}(\kappa)$. Here $L(\mathscr{S}) \subseteq U\left(\left\{\mathbb{Z}_{2}(\kappa)\right\}\right)$ because all accessible subrings of $\mathbb{Z}_{2}(\kappa)$ have cardinality $2^{\kappa}>\kappa$.

(e) This follows from Theorem 8 just as in (c) because $d\left(M_{n}\left(\mathbb{Z}_{2}\right)\left(\aleph_{0}\right)\right)=n$.

Acknowledgements. The research of the second author was supported by the University of Tasmania Mathematics Department Visitors Fund and also by NSERC grant No. 8789. Both authors appreciate the helpful comments of the referee.

\section{REFERENCES}

1. S. A. Amitsur, Prime rings having polynomial identities with arbitrary coefficients, Proc. London Math. Soc. 17 (1967), 470-486.

2. T. Anderson, K. KaArli and R. Wiegandt, Radicals and subdirect decomposition, Comm. Algebra 13 (1985), 479-494.

3. K. I. Beldar and K. Salavová, Some examples of supernilpotent nonspecial radicals, Acta Math. Acad. Sci. Hung. 40 (1982), 109-112.

4. G. M. Bergman and I. M. Isaacs, Rings with fixed point free group actions, Proc. London Math. Soc. 27 (1973), 69-87. 
5. S. Burris, Free algebras as subdirect products, Algebra Universalis 11 (1980), 133-134.

6. H. L. Chick and B. J. Gardner, The preservation of some ring properties by semilattice sums, Comm. Algebra 15 (1987), 1017-1038.

7. M. Cohen and S. Montgomery, Group graded rings, smash products, and group actions, Trans. Amer. Math. Soc. 282 (1984), 237-258.

8. N. Divinsky, Rings and Radicals (University of Toronto Press, Toronto, 1965).

9. J. W. Fisher and S. M. Montgomery, Semiprime skew group rings, J. Algebra 52 (1978), 241-247.

10. B. J. Gardner, Radical Theory (Longman/Wiley, Harlow/New York, 1989).

11. K. R. Goodearl, Prime ideals in regular self-injective rings II, J. Pure Appl. Algebra 3 (1973), 357-373.

12. K. R. Goodearl, Von Neumann Regular Rings (Pitman, London, 1979).

13. C. W. Koнts, Prime ideals in rings of continuous rings, Illinois J. Math. 2 (1958), 505-536.

14. L. C. A. van Leeuwen and T. L. Jenkins, A supernilpotent nonspecial radical class, Bull. Australian Math. Soc. 9 (1973), 343-348.

15. M. LoRenz. Finite normalizing extensions of rings, Math. Z. 176 (1981), 447-484.

16. M. Lorenz and D. S. Passman, Prime ideals in crossed products of finite groups, Israel $J$. Math. 33 (1979), 89-132.

17. E. Matlis, The minimal prime spectrum of a reduced ring. Illinois J. Math. 27 (1983), 353-391.

18. S. Montgomery, Prime ideals in fixed rings, Comm. Algebra 9 (1981), 423-449.

19. M. M. Parmenter and P. N. Stewart, Excellent extensions, Comm. Algebra 16 (1988), 703-713.

20. D. S. Passman, It's essentially Maschke's theorem, Rocky Mountain J. Math. 13 (1983), $37-54$.

21. Yu. M. Ryabukhin, Overnilpotent and special radicals, Studies in Algebra and Math. Anal., Izdat "Karta Moldovenjaske", Kishinev, 1965, 65-72.

22. Yu. M. Ryabuknin, Supernilpotent and special radicals, Algebra and Modules, Mat. Issled. 48 (1978), 80-93, 169.

23. L. H. ROweN, A subdirect decomposition of semiprime rings and its application to maximal quotient rings, Proc. Amer. Math. Soc. 46 (1974), 176-180.

24. R. L. Snider, Lattices of radicals, Pacific J. Math. 40 (1972), 207-220.

25. F. A. Sż̇sz, Radicals of Rings (Budapest, Akadémiai Kiadó, 1981).

26. R. WIEgand, Radical and Semisimple Classes of Rings (Queen's Papers in Pure and Applied Mathematics, No. 37, Kingston, 1974).

Mathematics Department

UNIVERSITY OF TASMANIA

Hobart, Tasmania 7001

Australia

\author{
Department of Mathematics, Statistics \\ and Computing Science \\ Dalhousie University \\ Halifax, Nova scotia \\ Canada B3H 3J5
}

